\title{
Surface temperature measurement and heat load estimation for carbon targets with plasma contact and machine protection
}

\author{
A. Herrmann \\ Max-Planck-Institut für Plasmaphysik, Boltzmannstr. 2, D-85748 Garching, Germany \\ E-mail: Albrecht.herrmann@ipp.mpg.de
}

\begin{abstract}
Surface temperature control of actively cooled targets is a part of the machine protection in long pulse experiments. The protection of the target is as good as the surface measurement and the thermal model of the target. This paper deduces thermal parameters necessary to correct the measured temperature by surface effects. The temperature measurement error due to the intrinsic CFC structure is discussed. Layers on top of the bulk material result in a change of the temperature response and in an overestimation of the calculated heat flux. All discussed effects did not reduce the machine safety but can reduce the operational space of the experiment. The thermal surface properties have to be checked in-situ by changing the heat load to the target and calculating the temperature response.
\end{abstract}

\section{Introduction and motivation}

Steady state fusion experiments rely on equilibrium between input power and power exhaust. Depending on the magnetic field configuration most critical structures are limiters or divertors. A plasma heating power of about $20 \mathrm{MW}$ in the limiter tokamak Tore Supra, 20 MW in the stellarator W7-X or $100 \mathrm{MW}$ in the next step divertor experiment ITER has to be received stationary. Actively cooled high heat flux components were developed and tested at Tore Supra for heat loads of about $10 \mathrm{MW} / \mathrm{m}^{2}$ [1]. The divertor concept for ITER aims at $10 \mathrm{MW} / \mathrm{m}^{2}$ maximum stationary heat load but can tolerate up to 300 cycles with $20 \mathrm{MW} / \mathrm{m}^{2}$ [2]. The divertor concept of the W7-X stellarator is specified to withstand a stationary heat flux up to $10 \mathrm{MW} / \mathrm{m}^{2}[3,4]$. Heat flux limiting components are brazed or welded structures inside the actively cooled targets. For $\mathrm{W} 7-\mathrm{X}$, the $10 \mathrm{MW} / \mathrm{m}^{2}$ standard load case results in a temperature in the $\mathrm{CuCrZr}-\mathrm{Cu}$ interface of $450^{\circ} \mathrm{C}$ or $70 \%$ of the maximum temperature [3], 
i.e. the safety margin is about 1.4 . In contrast, the corresponding surface temperature is below $1000{ }^{\circ} \mathrm{C}$, i.e. more than factor of 2 below the tolerable surface temperature of Carbon Fibre Composite (CFC).

Overloading of target tiles will, at the end, interrupt the cooling because no inherent safe technical concepts could be found. A safe machine operation requires a reliable and permanent control of the target heat load. This will be done by controlling the target surface temperature with requirements as defined e.g. in the ITER [5] and W7-X design documents [6]. But, the measured surface temperature is only a good figure for machine protection if a target in plasma contact has the same thermal properties as used for the model calculation and for the definition of the surface temperature limits.

Experiences from running experiments show that the assumption of an ideal target is violated and the thermal properties of the target surface are non-ideal. There are two main reasons for this. First, CFC has an intrinsic structure on a sub-millimeter scale which results in a non homogeneous surface temperature distribution. In addition, the manufacturing process of Fine Grain Graphite (FGG) and CFC damages the surface. Second, plasma target interaction can result in surface modifications and layer deposition. This paper discusses both effects with the focus on a route to identify parameters allowing to correct the measured temperature by the surface effects.

\section{Graphite without plasma exposure}

Carbon is used as target material for heat receiving structures because of its unique material properties (no melting, shock resistance, high sublimation temperature). Fine grain graphite (FGG) is commonly used in quasi stationary machines and carbon fibre composite (CFC) is needed for actively cooled structures due to the higher mechanical strength.

Thermographic measurements at carbon surfaces show that the temperature increase under heat loads is higher than expected from the effusivity $b=\sqrt{\kappa \rho c}$ of the bulk material. The 
effusivity is the capability of heat penetration and relevant for transient events where the temperature increase with a heat load $\mathrm{q}_{\mathrm{s}}$ is: $\Delta T_{s}=\frac{2}{\sqrt{\pi}} \frac{q_{s}}{b} \sqrt{t}$

This behaviour was found for virgin carbon $[7,8]$ as well as plasma exposed samples [9-11] in lab experiments and by plasma heating. A typical temperature response on a nearly square like heat pulse is shown in Fig. 1 for a plasma exposed surface and a polished part of the same target tile. On the time scale of the measurement $\left(\mathrm{t}_{\text {frame }}=500 \mu \mathrm{s}\right)$, the temperature increase is stepwise at the beginning of the pulse followed by an increase with the square root of time as expected from Equ. 1. Because the temperature jump is found not only for targets with plasma wall contact but also for virgin targets, it is supposed that this jump is due to grains with a reduced contact to the bulk. Carefully polishing can remove the loosely connected grains resulting in an ideal temperature behaviour of FGG [8]. The heat flux to the target can be estimated from the square root in time like behaviour at later times in the heat pulse, independent of the temperature jump. This value, $\mathrm{q}_{\mathrm{s}}=4.2 \mathrm{MW} / \mathrm{m}^{2}$ for the example of Fig. 1 , can be then used to estimate the heat transmission coefficient for the surface from the temperature jump, $\alpha=q_{s} / \Delta T \approx 140-200 \mathrm{~kW} / \mathrm{m}^{2} \mathrm{~K}$. This is equivalent to a thermal resistance of $\alpha^{-1} \approx 5 \div 8 \mathrm{~K} / \mathrm{MWm}^{-2}$. If the heat transmission coefficient for the used material is known, e.g. from laboratory experiments, the heat load to the target can be estimated from the temperature jump as well as the temporal evolution of the surface temperature. This allows a consistency check for the heat flux calculation and the detection of additional surface modifications.

The surface temperature distribution measured on CFC shows a much larger scatter during homogeneous heat load compared to FGG [7], as expected from the intrinsic structure of CFC consisting of fibres and filler (matrix) material with high and low heat conductivity, respectively. In addition fibres with lateral orientation can reduce the heat flux into the bulk. Thermally, CFC should be described, at least, as a two component system with different heat 
conductivity but comparable heat capacity. If these components are not coupled by lateral heat conduction the temperature ratio between the components is given by the effusivity ratio of the components (see equ. 1). Because the fibre has typically a 5-10 times higher heat conductivity [12], this temperature ratio is about 2-3. For higher temperatures the lateral heat conduction becomes significant and limits the maximum temperature difference at the surface. This difference can be estimated from the dimension of the component with bad heat conduction and the thermal properties by assuming that the lateral heat flux is in the same order as the heat flux into the bulk. With a typical lateral dimension of half the fibre diameter $(\mathrm{d}=100 \mu \mathrm{m})$ and a lateral heat conductivity which is a factor of 5 lower compared to the heat conductivity into the depth, i.e. about $\kappa=40 \mathrm{~W} / \mathrm{m} / \mathrm{K}$, the resulting temperature difference (thermal resistance) is $\Delta T / q=d / \kappa \approx 2.5 \mathrm{~K} / \mathrm{MWm}^{-2}$. A measurement of the temperature pattern at CFC NB-31 with high temporal and spatial resolution loaded with a laser beam of $10 \mathrm{MW} / \mathrm{m}^{2}$ over $10 \mathrm{~ms}$ [7] is shown in Fig. 2. Localized hot spots are found in addition to the fibre and the filler. The temperature behaviour of fibre, filler and most of the hot spots can be characterized as discussed for the FGG material. At the start of the heating pulse, a temperature jump is observed. Later in time, the temperature evolution follows the square root of time dependence with a heat conductivity of the fibre which is 5 times higher compared to the filler. The resulting thermal resistance for the filler and the fibre is comparable to that of the FGG surface $\left(8 \mathrm{~K} / \mathrm{MWm}^{-2}\right.$ and $6 \mathrm{~K} / \mathrm{MWm}^{-2}$ for the filler and the fibre, respectively). Hot spots result in a significant stronger local temperature increase dominated by a thermal resistance of about $30 \mathrm{~K} / \mathrm{MWm}^{-2}$, i.e. $300 \mathrm{~K}$ temperature excursion for the design heat load of $10 \mathrm{MW} / \mathrm{m}^{2}$ in $\mathrm{W} 7-\mathrm{X}$ and ITER. The fraction of hot spots is between 3 and $10 \%$ of the target area.

A temperature measurement with a few millimetre spatial resolution, as envisaged for machine protection systems, would integrate over all these temperature components. This will 
influence the accuracy of the surface temperature measurement. Because the temperature pattern is dominated by the intrinsic structure and the manufacturing of the CFC, the measurement error can be estimated by characterizing the surface behaviour of the used CFC and calculating a temperature correction curve as shown in the following for the measurement of Fig. 2.

The number of photons received by a millimetre sized detector pixel is the sum of the photons from the three different temperature regions with parameters as given in Table 1.

Table 1. Parameters of the CFC components.

\begin{tabular}{|c|c|c|c|}
\hline & fibre & filler & Hot spot \\
\hline Filling ratio & 0.45 & 0.45 & 0.1 \\
\hline Th. resistance & 6 & 8 & $30-50$ \\
\hline $\mathrm{K} / \mathrm{MWm}^{-2}$ & & & \\
\hline
\end{tabular}

This sum of photons from different temperature regions is then interpreted as caused by a uniform temperature in the field of view of a millimetre size detector. Fig. 3 shows the ratio between the bulk temperature (fibre without surface effects) and the temperature derived from the sum of all components. Parameters are the detection wavelength and the heating power. It is obvious, that for all parameter combinations the measured temperature is higher than the bulk temperature. From the viewpoint of machine protection, this is a safe situation. But, the mismatch between measured temperature and target temperature reduces the operational space for the machine, in particular in the near infra-red wavelength region $(1 \mu \mathrm{m})$. The relative contribution of the hot spot decreases with increasing wavelength because the change of the photon flux with temperature becomes lower (smoother Planck function). The mismatch increases with increasing heat flux due to the heat flux dependent temperature jump (see Table 1.). The dashed curve in Fig. 3 is the $10 \%$ error line. It is obviously that all but the 
measurement in the near ir-region at $10 \mathrm{MW} / \mathrm{m}^{2}$ has an error below $10 \%$. Such an error is inside the safety margin of 1.4 and comparable to the temperature error due to a change of emissivity in the LWIR region $(10 \mu \mathrm{m})$.

The parameterization of the thermal surface properties of CFC material used for target materials on a sub-millimetre scale can be used to calculate a correction curve which allows calculating the bulk temperature without surface effects from the measured temperature by single step iteration. $1^{\text {st }}$ step, calculate the heat load to the target with averaged thermal parameters for the bulk and the surface. $2^{\text {nd }}$ step, use this heat flux and the parameterization to calculate the real bulk temperature. The required parameters can be gained during the acceptance test of the target or in-situ by measurements with spectral resolution [13-15]

\section{Plasma effects}

In addition to the intrinsic surface temperature structure, plasma target contact can result in an additional modification of the surface. These surface modifications might be caused by ion implantation or layer deposition. How to consider these effects into the estimation of the true bulk temperature and the heat flux calculation model depends on the layer thickness and its thermal contact to the bulk material. The combination of the thermal layer thickness and contact o the bulk material allows distinguishing for limits.

(i) A thin layer with good heat contact has a low heat capacity and the temperature distribution becomes stationary at time scales on the order of the frame rate of the measurement. A stationary temperature profile can be considered by a thermal resistance in the same way as for the intrinsic surface effects. The measured time evolution of temperature is that of the bulk (see Fig. 1).

(ii) A thick layer with good heat contact shows two time scales, the time evolution of the layer temperature at the start of the heating and the heating of the bulk at later times [see also[11]]. 
(iii,iv) The temperature of a layer with bad heat contact is comparable to a thin target with a cooling at the backside via a heat transmission edge condition. The surface temperature is determined by the heat capacity and the accumulated energy and increases linear in time until radiation losses becomes significant at very high temperatures. The ratio of heating power and radiated power determines whether or not such a layer evaporates or not.

To estimate layer effects on the temperature measurement and finally the heat flux calculation, the temporal temperature evolution for a target consisting of the bulk, an interlayer with a heat resistance of $\alpha^{-1} \approx 7.7 \mathrm{~K} / \mathrm{MWm}^{-2}$ and a $30 \mu \mathrm{m}$ thick top layer with different thermal parameters (see Table. 2) were calculated with the FE code FlexPDE [16]. This calculated temperature evolution was then used as input for heat flux calculation with a thermal model for a bulk with thermal resistance on top. Fig. 4 shows the calculated heat flux for 4 parameter sets. In case 1 the model for the temperature calculation and the heat flux calculation are the same, i.e. the curve represents really applied heat flux. Case 2 represents a layer with the same thermal properties as the bulk. The slower increase of the heat flux is because the temperature jump is delayed by the time the heat needs to diffuse through the layer. For a layer with lower heat capacity this transfer time is short and again a jump like temperature increase is observed (case 5). The calculated heat flux is higher due to the additional temperature drop across the layer caused by the lower heat conductivity. Increasing the heat capacity and keeping the lower heat conductivity reduces the temperature slope as in case 2 but now again with a higher calculated heat flux (case 4). The temporal temperature behaviour at the end of the heating is qualitatively the same as at the raising edge. It should be mentioned, that the accumulated energy is independent on the thermal model for the surface because it is dominated by the bulk properties (Fig. 4). 
Table 2 Thermal parameters of the top layer as used for the calculation of the surface temperature.

\begin{tabular}{ccc}
\hline case & $\rho \mathrm{c} / \mathrm{MJ} / \mathrm{m}^{3} \mathrm{~K}$ & $\kappa / \mathrm{W} / \mathrm{m}^{2}$ \\
\hline 1 & NO top layer \\
2 & 1.08 & 110 \\
4 & 1.08 & 22 \\
5 & 0.108 & 22 \\
\hline
\end{tabular}

\section{Conclusions}

Surface temperature control of actively cooled targets is a part of the machine protection in long pulse experiments. The protection of the target is as good as the surface measurement and the thermal model. Measurements in existing machines have shown that the surface temperature measurement is influenced by the intrinsic in-homogeneous structure of the CFC material and layers deposited by plasma target interaction. The surface effect for FGG and $\mathrm{CFC}$ can be parameterized by thermal resistances. For CFC at least three different thermal resistances are required. Using these parameters a correction curve can be calculated which allows to deduce the true bulk temperature from the measured surface temperature. The required parameters can be gained during the acceptance test of the target or in-situ by measurements with spectral resolution [13-15]. Layers in top of the bulk material result in a change of the temperature response and in an overestimation of the calculated heat flux. All discussed effects did not reduce the machine safety but can reduce the operational space of the experiment. The thermal surface properties can be checked in-situ by changing the heat load to the target and calculating the temperature response. Temperature monitoring as tool for target protection must include heat flux calculations. 


\section{Figures}

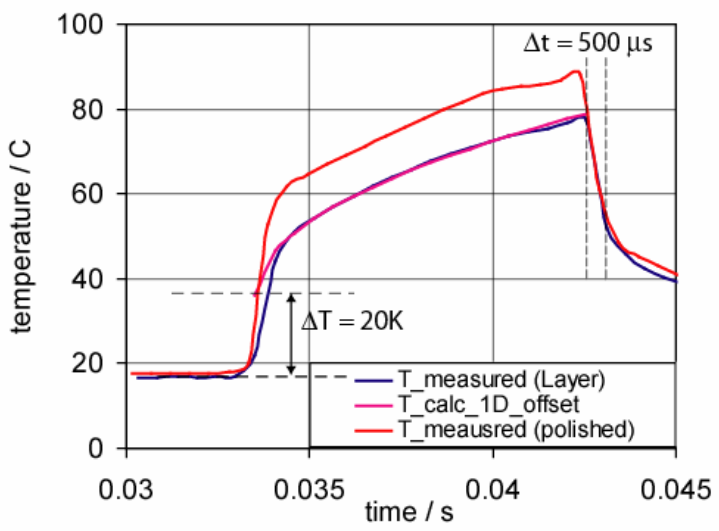

Fig. 1. Temporal evolution of the surface temperature of FGG during a laser pulse of $10 \mathrm{~ms}$ measured at an upper divertor tile of ASDEX Upgrade (S8, A1). The lower curve was measured at a plasma exposed part, the upper curve (polished) on a position that was polished by sandpaper. The experimental arrangement of ir-camera and laser parameters are described in [7].
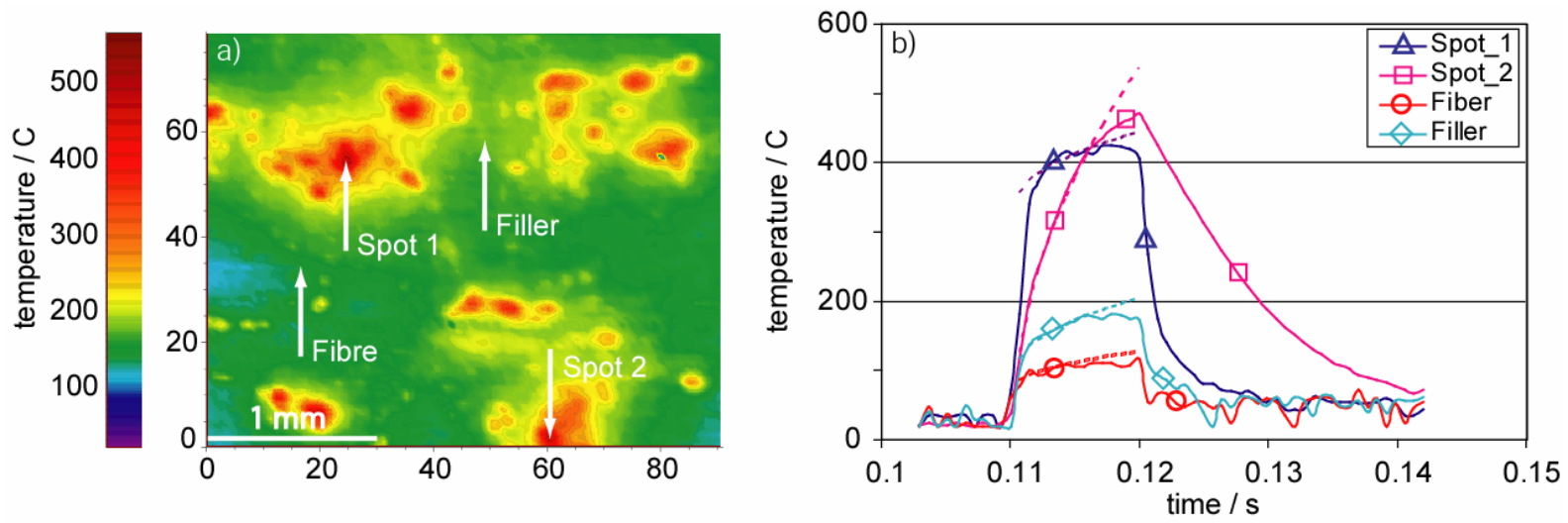

Fig. 2 a) Temperature pattern with $30 \mu \mathrm{m}$ spatial resolution at the end of an heat pulse $\left(10 \mathrm{MW} / \mathrm{m}^{2}, 10 \mathrm{~ms}\right)$. b) Temporal evolution of the surface temperature at different locations as marked in a). The size of the 2D frame corresponds to the typical spatial resolution of thermography systems for machine protection and physics investigations. The heat conductivity of the fiber is a factor of 5 better than that of the filler material. 


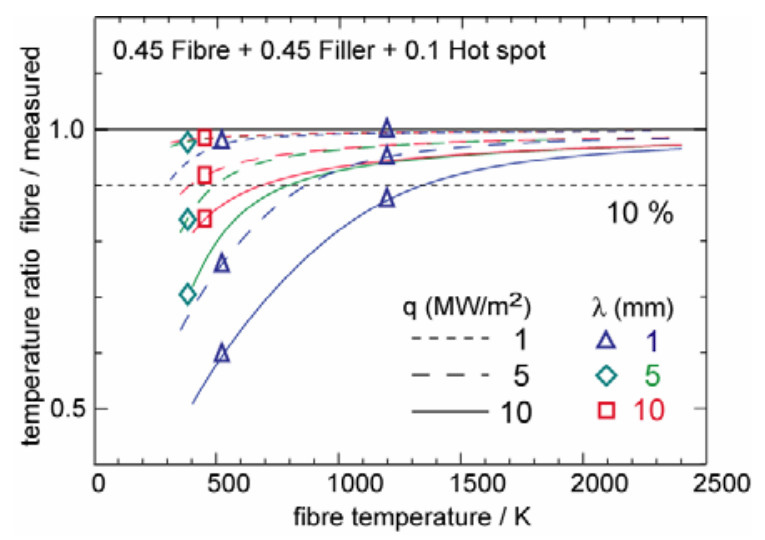

Fig. 3 Temperature ratio - true temperature of the fibre material compared to the temperature derived from the mixing of photons from different temperature regions of the target.

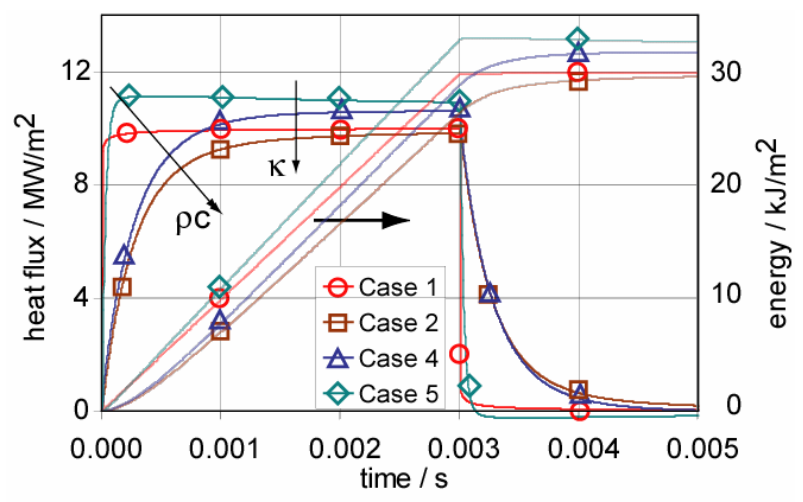

Fig. 4 Heat flux calculated with the thermal model of a bulk material with a thermal resistance on top and different temperature evolutions calculated for different layers on top as input (parameters - see Table 2).

\section{References}

[1] Grosman A 2005 Fusion Eng. Des. 74 49-57

[2] Merola M, Danner W and Pick A 2005 Fusion Eng. Des. 75-9 325-331

[3] Boscary J, Greuner H, Mendelevitch B, Schedler B, Scheiber K, Schlosser J and Streibl B 2005 Fusion Eng. Des. 75-9 451-455

[4] Renner H, Boscary J, Greuner H, Grote H, Hoffmann F W, Kisslinger J, Strumberger E and Mendelevitch B 2002 Plasma Phys. Control. Fusion 44 1005-1020

[5] ITER - Project Integration Document (PID); https://users.iter.org/users/idm?document_id=ITER_D_2234RH, 2004.

[6] W7-X design review 2005

[7] Herrmann A, Balden M, Bohmeyer W and Hildebrandt D 2004 Phys. Scrip. T 111 98100

[8] Hildebrandt D, Naujoks D and Sunder D 2005 J. Nucl. Mater. $337-39$ 1064-1068 
[9] Herrmann A and ASDEX Upgrade Team 2001 Europhysics Conference Abstracts (CD-ROM, Proc. of the 28th EPS Conference on Controlled Fusion and Plasma Physics, Madeira 2001) 25A 2109-2112

[10] Andrew P, Coad J P, Eich T, Gauthier E, Herrmann A, Matthews G F, Riccardo V and Stamp M 2003 J. Nucl. Mater. 313 135-139

[11] Andrew P, Coad J P, Corre Y, Eich T, Herrmann A, Matthews G F, Paley J I, Pickworth L, Pitts R A and Stamp M F 2005 J. Nucl. Mater. 337-339 99-103

[12] Pestchanyi S and Landman I 2006 Fusion Eng. Des. 81 275-279

[13] Reichle R, Pocheau C, Balorin C, Delchambre E, Desgrange C, Guilhem D, Henry S, Messina P and Roche H 2004 Review of Scientific Instruments 75 4129-4132

[14] Reichle R, Pocheau C, Delchambre E, Ducobu L, Faisse F, Guilhem D, Jouve M, Moulin D, Roche H and Thomas E 2003 J. Nucl. Mater. 313-316 711-715

[15] Delchambre E, Reichle R, Mitteau R, Missirlian M and Roubin P 2005 J. Nucl. Mater. 337-339 1069-1072

[16] FlexPDE. http://www.pdesolutions.com/. 